\title{
A COMPARATIVE STUDY ON ELECTROPLATING OF FDM PARTS
}

\author{
Azhar Equbal $^{1}$, Md. Israr Equbal ${ }^{2}$, Anoop Kumar Sood $^{3}$, Md. Asif Equbal ${ }^{4 *}$ \\ ${ }^{1}$ Department of Manufacturing Engineering, National Institute of Foundry and Forge Technology, \\ Ranchi 834003, India \\ ${ }^{2}$ Department of Mechanical engineering, Aurora's technological and research institute, Uppal, \\ Hyderabad 500098, India \\ ${ }^{3}$ Department of Manufacturing Engineering, National Institute of Foundry and Forge Technology, \\ Ranchi 834003, India \\ ${ }^{4}$ Department of Mechanical Engineering, Cambridge Institute of Technology, Ranchi 831005, India
}

(Received: January 2017 / Revised: September 2017 / Accepted: October 2017)

\begin{abstract}
Electroplating on fused deposition modeling parts through two different routes is presented in the study. One route follows the conventional method of electroplating using chromic acid for surface preparation or etching and the other route uses the novel method of electroplating using aluminium charcoal (Al-C) paste for surface preparation. Same plating conditions are used for both the routes employed. The result proposes that instead of shell cracking in few electroplated samples, Al-C route is also capable of producing good copper deposition on FDM samples. Cracks may develop in few samples electroplated through Al-C route, because of dissolution of paste at high operating condition during electroplating. Proper drying of electrolessly plated samples and adaptation of suitable operating condition reduces the risk of electroplated shell cracking.
\end{abstract}

Keywords: Cracking; Deposition; Electroplating; Etching; Surface preparation

\section{INTRODUCTION}

In electroplated parts properties of both metal and plastic can be achieved. These parts are provided with many attractive properties like lightweight, corrosion resistance, conductivity and abrasion resistance. Electroplated products found uses in varieties of industrial applications including automotive industries, electronic industries and domestic fitting applications. Electroplating can be done both on metal and plastic parts. Electroplating of metal parts is common. Plastic parts are non-conductive and hence their electroplating is not simple. Plating of plastic parts is done by the process known as metallization. Metallization is the process in which a coated layer of metal is provided over plastic parts. Metallization is of two types: primary metallization and secondary metallization. Primary metallization induces a thin layer of metallic coating on the plastic parts whereas secondary metallization is done to increase the thickness of primary metallized layer.

Primary metallization is done using various methods like electroless plating, brushing of metallic paint, metal spraying, dipping in a metal paint, sputtering and vacuum metallization (Radulescu et al., 2002; Equbal et al., 2015). Every process has its own merits and demerits in terms of

\footnotetext{
* Corresponding author's email: equbal.asif@yahoo.com, Tel: +91-651-6566121, Fax: +91-651-2265450 Permalink/DOI: https://doi.org/10.14716/ijtech.v8i5.875
} 
economy, user friendly applications and complexity. For secondary metallization, electroplating is most commonly used. Conventional method of metallization on FDM (fused deposition method) parts uses chromic acid which is dangerous and produces health hazards. To prevent the problems related with chromic acid alternative method is desired. The present paper thus uses a novel method of electroplating that uses Al-C paste for electroless plating and it is followed by electroplating for secondary metallization. To validate the present method, results of electroplating process is also compared with result of conventional chromic acid metallization.

FDM is 3D (Three dimensional) printing processes which build the part using layer by layer deposition principle directly from the part digital information. The process needs no specific process plan and no special tooling is required. The detail of the process can be found elsewhere in literature (Equbal et al., 2015; Sood et al., 2009; Sood et al., 2010). FDM uses ABS (acrylonitrile butadiene styrene) as the part material and ABS has got excellent metallization properties (Kuzmik et al., 1990). Electroless plating is widely used for metallization of ABS plastic. This process doesn't require any electrical support and has the advantage of providing uniform plating even in sharp corner without build up (Hanna et al., 2004; Xu et al., 2004). Normal electroless process is a multi-step process using long deposition time and chemicals that are costly and environmentally hazardous. To eliminate this, number of researchers suggested the use of less costly and environmental friendly chemicals. In this direction, use of Al-C paste is suggested (Li \& Yang, 2009). Al-C paste method is a novel method for electroless plating process but the practicality of their process is not tested with electroplating. As the process of electroplating has its own process variables and settings, the feasibility of Al-C paste method needs to be tested. Thus in this paper, electroplating using Al-C paste route is used and compared with the normal industrial electroplating method which uses chromic acid for surface preparation.

\section{EXPERIMENTAL}

Part fabrication is done using fused deposition modelling (FDM) by Stratasys Inc., USA. ABS P400 is the part material that is used for part fabrication (Lustraflex material safety data sheet, 2009). The part used in this experimental study is of cylindrical shape. Copper $(\mathrm{Cu})$ is used for metallization. Copper is selected for its low cost and excellent conductivity (Li \& Yang, 2009). $\mathrm{H}_{2} \mathrm{SO}_{4}$ is used as an electrolyte. For conventional chromic acid plating different chemicals like chromic acid, sulphuric acid, sodium sulphite, palladium/tin and ethylenediaminetetraacetic acid disodium (EDTANa 2 ) is used. Al-C method uses fine aluminium metal powder, charcoal granules, enamel paint, distilled water, 320 grit sand paper and brushes. Different equipment used in experiments is magnetic stirrer of 2-liter capacity (Remi) and electroplating setup (Rectifier, plating tank and number of hoses).

\subsection{Normal Electroless Process}

The normal electroless method consists of multiple stages as described below. The steps involved are as follows:

\section{Cleaning:}

ABS parts were cleaned using pumice powder and scoured with 320 grit sandpaper to remove oil, dirt, grease etc. and also to increase surface area by developing micro-cavities.

\section{Etching:}

Etching is an important phase for achieving good metal plastic bond (Nicolas-Debarnot et al., 2006; Wang et al., 2007; Di et al., 2011). Cleaned ABS parts were dipped in an aqueous solution containing chromic acid $(600 \mathrm{~g} / \mathrm{l})$, Sulphuric acid $(150 \mathrm{ml} / \mathrm{l})$ and deionised water, maintained at $60{ }^{\circ} \mathrm{C}$ for 10-15 minutes. The samples were then taken out and washed 2-3 times carefully. 


\section{Neutralization:}

Residual amount of chromium remaining in the ABS surface was removed with a sodium sulphite as a reducing agent to prevent its inhibition in further steps because trace amounts of chromium may also completely inhibit electroless deposition. The parts were dipped in the solution of 10 $\mathrm{g} / \mathrm{l}$ of Sodium sulphite at $25^{\circ} \mathrm{C}$ for about 2 minutes and washed with water.

\section{Activation:}

An activator consisting of colloidal suspension of palladium/tin $(\mathrm{Pd} / \mathrm{Sn})$ catalyst powder was applied over conditioned part surface (O'Kelly et al., 2000; Hong et al., 2002). During experimentation samples were activated at $40^{\circ} \mathrm{C}$ for 7 minutes and finally washed with water.

\section{Acceleration:}

It dissolves excess $\mathrm{Sn}$ and removes it from the surface for exposing the adsorbed Pd. The samples are dipped in a solution mixture containing $30 \mathrm{~g} / \mathrm{l}$ sodium hydroxide $(\mathrm{NaOH}), 3 \mathrm{~g} / \mathrm{l}$ copper sulphate $\left(\mathrm{CuSO}_{4}\right)$ and $15 \mathrm{~g} / \mathrm{l}$ ethylenediaminetetraacetic acid disodium (EDTANa $)$ at $55^{\circ} \mathrm{C}$ for about 7 minutes. The samples were finally washed with water followed by acidic bath treatment.

\section{Electroless deposition:}

In this step, electroless plating is carried out in electroless bath prepared by adding $5 \mathrm{wt} \%$ of copper sulphate $\left(\mathrm{CuSO}_{4}\right)$ and $15 \%$ of sulphuric acid $\left(\mathrm{H}_{2} \mathrm{SO}_{4}\right)$. The deposition was done at room temperature for $48 \mathrm{hrs}$.

\subsection{Preparation of Al-Charcoal Paste and Plating of ABS Parts}

Aluminium powder, charcoal, enamel and distilled water were mixed at a weight ratio of 40:3:36:21 in a $200 \mathrm{ml}$ beaker. The prepared mixture was then stirred in a magnetic stirrer vigorously till it forms paste. The paste was then applied carefully with a brush on ABS parts precleaned with soap and distilled water. ABS parts are then allowed to dry completely at room temperature. Al-C pasted samples ate then scoured with 320 grit sandpaper and rinsed well with distilled water. Electroless bath was prepared by adding $5 \mathrm{wt} \%$ of copper sulphate $\left(\mathrm{CuSO}_{4}\right)$ and $15 \%$ of sulphuric acid $\left(\mathrm{H}_{2} \mathrm{SO}_{4}\right)$. The deposition was done at room temperature for $48 \mathrm{hrs}$ which also eliminate the need of Cole-Parmer StableTemp digital hot plate (Vernon Hills, Illinois) as used by Li and Yang (2009). It is to be noted that the concentration of bath used by Li and Yang, was $15 \mathrm{wt} \% \mathrm{CuSO}_{4}$ and $5 \mathrm{wt} \%$ of $\mathrm{H}_{2} \mathrm{SO}_{4}$. Initially same bath was used but the result obtained was not satisfactory as it was done at normal room temperature. Thus elevated temperature and controlled environment was used.

\subsection{Electrical Performance Measurement, SEM and EDS}

Digital multimeter (VOLTCRAFT M-3850) was used for measuring the resistance of the copper plated FDM parts. The resistance was measured at 30 different points on the different surfaces and average was taken. The average resistance $(\bar{R})$ value together with standard deviation $(\sigma)$ was calculated according to Equations 1 and 2.

$$
\begin{gathered}
\bar{R}=\frac{\sum_{i=1}^{n} R_{i}}{n} \\
\sigma=\frac{\sqrt{\sum_{i=1}^{n}\left(R_{i}-\bar{R}\right)^{2}}}{n-1}
\end{gathered}
$$

where, $R_{i}$ is measured resistance value at $i^{\text {th }}$ point and total number of points are $n$. 
Adhesion assessment on electrolessly deposited Al pasted FDM samples was performed by standard tape test method using ASTM D 3359-02 (ASTM D3359, 2010). Tape test was used to evaluate the proper adherence of the Al-C paste over of ABS parts. The test was done only for Al-C pasted parts. During scouring of Al-C pasted parts, presence of aluminium seeds is only insured by presence of paste. The test was not required for normal electroless route since this does not involve any paste. Moreover the activator $(\mathrm{Pd} / \mathrm{Sn}$ catalyst powder) got deposited only in the micro-cavities formed during etching stage and further deposition is possible only because of these catalysts.

A ZEISS EVO-MA10 SEM scanning electron microscope coupled with an energy dispersive Xray spectrometer was used to examine the elemental composition and appearance of the copper in samples prepared by both individual methods.

\subsection{Electroplating}

It is a chemical deposition process in which electrical current is used to deposit thick metallic coating onto another conductive surface. It is done to provide useful materials with improved mechanical, decorative, electrochemical, electrical, magnetic or optical properties (Vagramyan, 1970). Electroplating not only enhances the look of a part but also produces a hard, durable surface and increases the strength of an electrolessly plated part. The complete electroplating process consists of number of chemicals and equipment. The electrolytic tank is connected to two filters: plate and cartridge filter. Plate filter consists of polypropylene layer for filtering the heavier and bigger impurities. Cartridge filter is also polypropylene filter in form of thread for separating the smaller impurities remained after passing through plate filter. These two filters are connected to electrolytic tank for continuous filtration of the electrolytic solution which is recirculated between filters and electrolytic tank. The rectifier provides the current density and voltage to the electroplating setup. The chemicals and their composition used in electrolytic bath are given in Table 1.

Table 1 Bath concentration used in electroplating of copper

\begin{tabular}{cc}
\hline Composition & Concentration \\
\hline $\mathrm{CuSO}_{4}$ & $200 \mathrm{gm} / \mathrm{lit}$ \\
$\mathrm{H}_{2} \mathrm{SO}_{4}$ & $60 \mathrm{ml} / \mathrm{lit}$ \\
$\mathrm{HCl}$ & $120 \mathrm{ml} / \mathrm{lit}$ \\
\hline
\end{tabular}

When the potential difference is applied copper from copper plate (anode) dissolves into the medium in form of $\mathrm{cu}^{2+}$ ions. Since the substrate or primary metallized $\mathrm{Cu}$ part is negatively charged, it accepts the copper ions and copper ions start depositing in the part and thereby building the $\mathrm{Cu}$ layer. The reactions are:

$$
\begin{aligned}
& \text { At Anode } \mathrm{Cu} \rightarrow \mathrm{Cu}^{2+}+2^{\mathrm{e} 1-} \\
& \text { At Cathode } \mathrm{Cu}^{2+}+2^{\mathrm{el}} \rightarrow \mathrm{Cu}
\end{aligned}
$$

In our study, the optimized condition was obtained at $2.5 \mathrm{Amp} / \mathrm{dm}^{2}$. Plating was done for 3 hours to ensure visible and thick deposition of copper layer on the electrolessly processed FDM parts. For both methods, composition and operating conditions are same. Figure 1 shows the schematic diagram of complete electroplating process. 


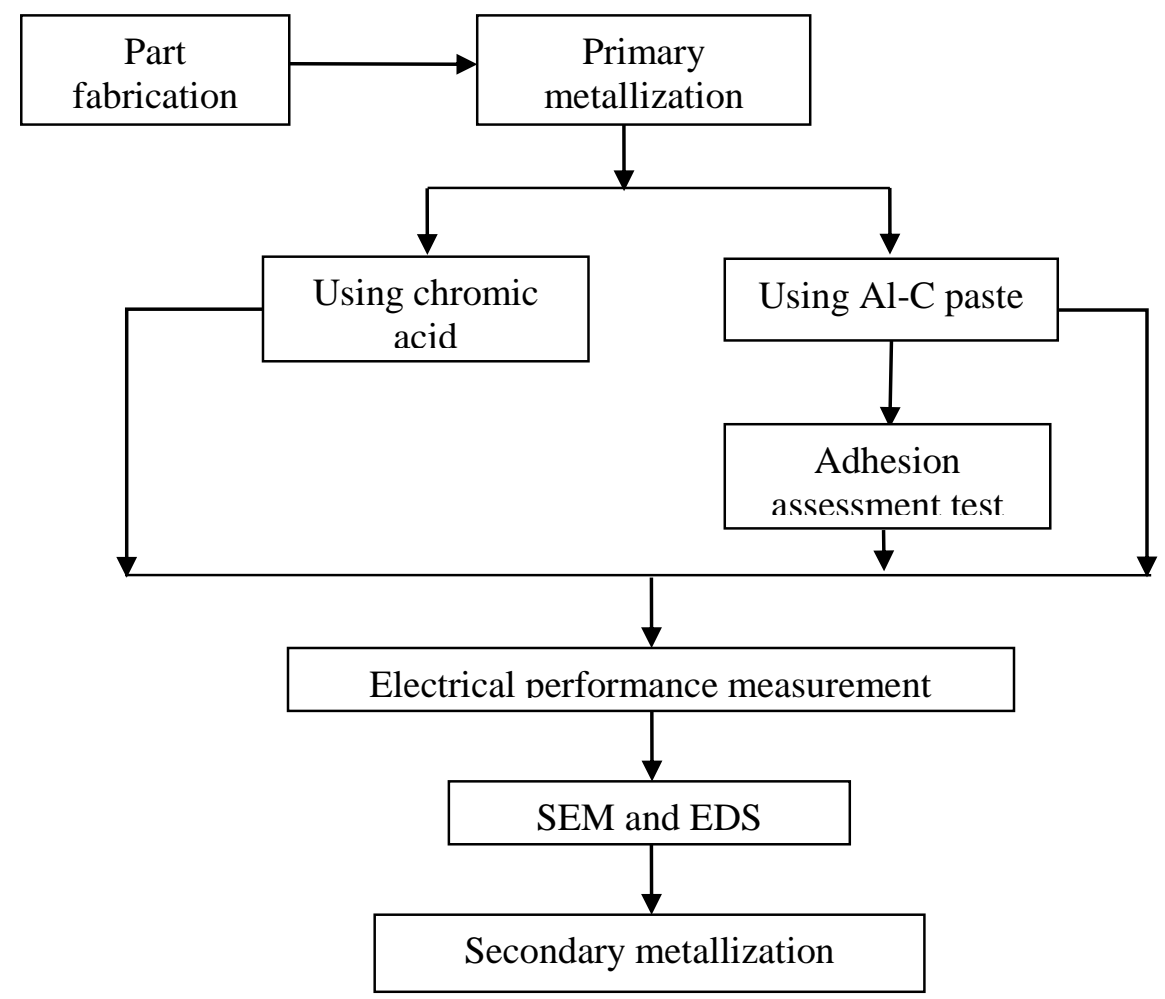

Figure 1 Schematic diagram of metallization processes

\section{RESULTS AND DISCUSSION}

The resistance value of the electrolessly plated part was measured by multimeter and standard deviation was calculated. Average resistance $(\bar{R})$ values together with standard deviation $(\sigma)$ obtained after electroless copper deposition for chromic acid etched samples and Al-C pasted at room temperature for $1 \mathrm{hr}, 24 \mathrm{hr}$ and $48 \mathrm{hr}$ of deposition time are presented in Table 2 and Table 3.

Table 2 Electrical performance of chromic acid etched samples

\begin{tabular}{ccc}
\hline \multirow{2}{*}{$\begin{array}{c}\text { Deposition time } \\
\text { (Hour, hr) }\end{array}$} & \multicolumn{2}{c}{ Acidic bath } \\
\cline { 2 - 2 } & $\overline{\mathrm{H}_{2} \mathrm{SO}_{4}}$ & $\sigma$ \\
\hline 1 & $\bar{R}(\mathrm{M} \Omega)$ & \\
24 & $>$ & $>$ \\
48 & 1.36 & 0.14 \\
\hline
\end{tabular}

"> denotes readings that are beyond the maximum measuring range (100 M $\Omega$ ) of the multimeter; "-" denotes there is no conductivity.

Table 3 Electrical performance of Al-C pasted samples

\begin{tabular}{ccc}
\hline \multirow{2}{*}{$\begin{array}{c}\text { Deposition time } \\
\text { (Hour, hr) }\end{array}$} & \multicolumn{2}{c}{ Acidic bath } \\
\cline { 2 - 2 } & $\bar{R}(\Omega)$ & $\sigma$ \\
\hline 1 & - & \\
24 & 0.11 & - \\
48 & 0.09 & 0.03 \\
\hline
\end{tabular}

"> " denotes readings that are beyond the maximum measuring range (100 M $\Omega)$ of the multimeter; "-" denotes there is no conductivity. 
It was observed that resistance value decreases with time showing the improvement in conductivity of part. It can be clearly observed from Table 2 and Table 3 that better conductivity was achieved after $48 \mathrm{hrs}$ of deposition time. It was noticed that after $48 \mathrm{hrs}$ of deposition, blue copper sulphate crystals spread over part surface creating its own layer and no conductivity was noted. Best conductivity was thus obtained at 48 hours of deposition time. It is also observed that the resistance values are in ohms for Al-C samples when compared with samples of conventional chromic acid metallization method. This means that the result obtained after electroless plating is better for the Al-C paste route than the conventional chromic acid route.

As good conductivity was achieved after $48 \mathrm{hrs}$ of deposition, SEM images are presented for 48 hrs of deposition time. Figure 2 present the SEM image, EDS mapping and EDS spectra of $\mathrm{Cu}$ deposition on samples of conventional chromic acid route after electroless $\mathrm{Cu}$ deposition in $\mathrm{H}_{2} \mathrm{SO}_{4}$ bath at room temperature. Figure 2a is the SEM image in which large $\mathrm{Cu}$ crystals can be clearly observed. EDS image presented in Figure $2 b$ shows copper distribution in the sample. Figure $2 \mathrm{c}$ presents the elemental analysis showing the presence of $88.83 \% \mathrm{Cu}, 5.39 \% \mathrm{O}, 0.78 \%$ $\mathrm{C}$ and $5 \%$ beryllium.
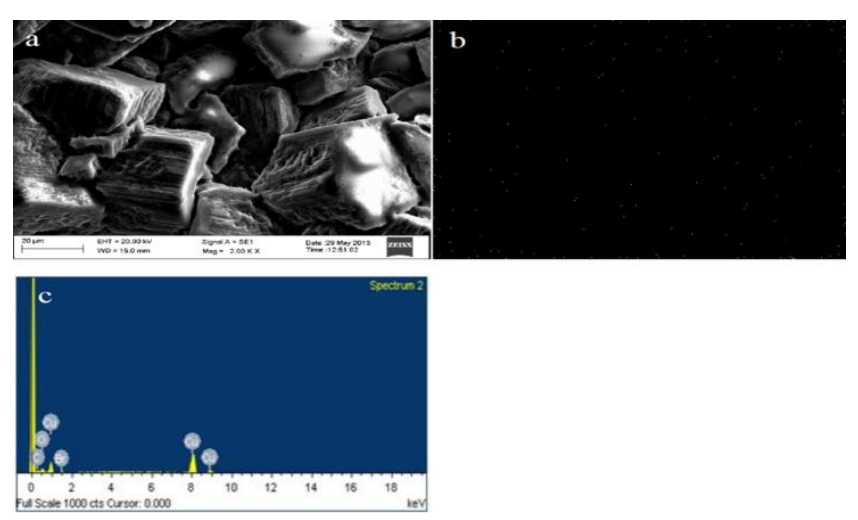

Figure 2 (a) SEM image; (b) EDS mapping; (c) EDS spectra of conventional route after electroless copper deposition in $\mathrm{H}_{2} \mathrm{SO}_{4}$ after $48 \mathrm{hrs}$

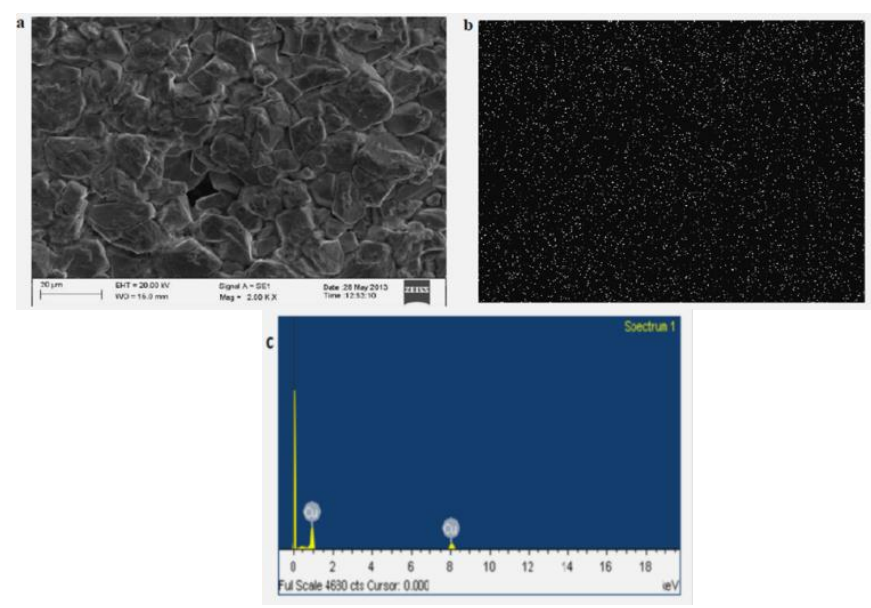

Figure 3 (a) SEM image; (b) EDS mapping; (c) EDS spectra of Al-C pasted samples after electroless plating in $\mathrm{H}_{2} \mathrm{SO}_{4}$ bath after $48 \mathrm{hr}$ of deposition time

Figure 3 presents the SEM image, EDS mapping and EDS spectra of $\mathrm{Cu}$ deposition on Al-C pasted samples after electroless $\mathrm{Cu}$ deposition in $\mathrm{H}_{2} \mathrm{SO}_{4}$ bath at room temperature after $48 \mathrm{hr}$ of deposition time. $\mathrm{Cu}$ crystals are closely connected and provide smooth appearance as shown in 
Figure 3a. EDS mapping presented in Figure $3 \mathrm{~b}$ further justify this showing $\mathrm{Cu}$ distribution after $48 \mathrm{hr}$ of deposition time. EDS mapping in Figure $3 \mathrm{c}$ shows that copper crystals are widely spread over the whole area of the sample covered in SEM. EDS spectra presented the peaks indicating copper at that region and also elemental composition was 100 atomic $\%$ copper. The reason for more copper deposition in the Al-C pasted samples is due to uniform distribution of $\mathrm{Al}$ in the paste and application of this paste to FDM parts. As the $\mathrm{Cu}$ gets deposited over the $\mathrm{Al}$ seeds exposed during etching and its growth increases with the deposition time, uniform $\mathrm{Cu}$ deposition is obtained after $48 \mathrm{hrs}$ of deposition time.

Adhesion evaluation of the entire Al-C pasted samples is done according to the scale defined by ASTM D 3359-02. All the ABS test samples after tape test shows no flaking and yield results in a scale of $4 \mathrm{~B}$ or $5 \mathrm{~B}$, meaning excellent adhesion strength. Finally, electroplating of samples is then done and compared.

Few cylindrical electroplated FDM parts obtained after electroplating using chromic acid for etching at given parameters are shown in Figure 4. It can be clearly seen that copper deposited over FDM parts are very good and uniform despite having the resistance values in $M \Omega$ after electroless deposition. To determine the thickness of the deposited copper layer, diameter of electroplated electrode was subtracted from raw ABS cylindrical part. The thickness was measured at different point and compared with raw ABS part and the average value was of thickness was calculated as $0.153 \mathrm{~mm}$.

\section{\#1}

Figure 4 Electroplated cylindrical ABS part using chromic acid for etching

Figure 5 and Figure 6 show copper plated FDM parts using Al-C paste method. Different steps used in normal electroless plating are eliminated in this process making it cost effective and time saving.

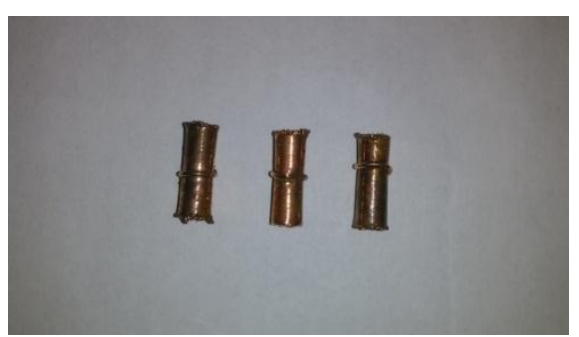

Figure 5 Electroplated cylindrical ABS part using Al-C paste

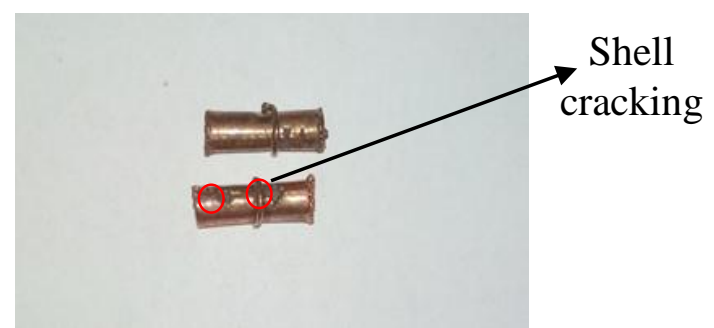

Figure 6 Electroplated ABS part using Al-C route showing shell cracking

Good and comparable copper deposition can be observed on the cylindrical ABS parts plated using Al-C paste. The thickness of deposition was found to be around $0.145 \mathrm{~mm}$ on average which shows good and comparative deposition when compared with chromic acid electroplated sample. The only problem observed was shell cracking at some regions in few samples and nonuniform deposition at edges. To overcome the problem of shell cracking proper drying of Al-C plated sample was performed and it was observed that the shell cracking is greatly reduce and it 
was hardly observed on any of electroplated samples. Moreover, the thickness of coating also marginally improved and found in the range $0.145-0.147 \mathrm{~mm}$.

\section{CONCLUSION}

The present paper compares two different routes of copper metallization for electroplating on FDM parts. These routes are: deposition method using chromic acid and a novel method of deposition using Al-C paste. This deposition was further confirmed with the conductivity via resistance value and SEM with EDS images. Important conclusions drawn from the study are: (1) Copper deposition is achieved through both the routes used; (2) In Al-C method, resistance was obtained in all the measured points but resistance values were not obtained at all the measured points in chromic acid route (Equbal et al., 2014); (3) After electroless deposition stage Al-C plated samples shows the best performance when compared with chromic acid route both in terms of electrical conductivity and $\mathrm{Cu}$ deposition. The reason for the better conductivity in $\mathrm{Al}-\mathrm{C}$ samples is uniform distribution of aluminium present in the paste applied. $\mathrm{Cu}$ crystal gets deposited in the region where $\mathrm{Al}$ seeds were present and the growth of $\mathrm{Cu}$ becomes denser with increase in the deposition time. Al used in paste for surface preparation itself acts as catalyst in the electroless $\mathrm{Cu}$ deposition process; (4) The resistance obtained for chromic acid route is higher (in $\mathrm{M} \Omega$ compared to $\Omega$ in Al-C route). The resistance is higher for this route because deposition occurs only in the micro-cavities formed during etching. During surface preparation stage microcavities are formed by removal of softer butadiene from ABS parts and increasing the surface area. It is in these micro-cavities where catalyst $(\mathrm{Pd} / \mathrm{Sn})$ is absorbed during activation. The etching may not be able to create cavities throughout the part which leads to non-uniform or localized deposition of $\mathrm{Cu}$ leading to higher resistance; (5) Conductivity varies because of non-uniform distribution and different sizes of $\mathrm{Cu}$ in each route. Also, conductivity improves with the deposition time; (6) After electroplating good and uniform copper deposition occurs in chromic acid etched samples despite of such poor conductivity obtained after electroless plating stage; (7) Al-C plated samples provide good deposition of copper after electroplating at the same operating conditions only with the problem of shell cracking in few samples; (8) Proper drying of Al-C samples reduces the problem of shell cracking.

\section{REFERENCES}

ASTM Designation, D3359-09, 2010. Standard Test Methods for Measuring Adhesion by Tape Test, pp.1-8

Di, L.Z., Liu, B., Song, J., Shan, D., Yang, D.-A., 2011. Effect of Chemical Etching on the Cu/Ni Metallization of Poly (Ether Ether Ketone)/Carbon Fiber Composites. Applied Surface Science, Volume 257(9), pp. 4272-4277

Equbal, A., Equbal, A., Sood, A.K., 2014. Metallization on FDM Processed Parts using Electroless Procedure. Procedia Materials Science, Volume 6, pp. 1197-1206

Equbal, A., Sood, A.K., 2015. Investigations on Metallization in FDM Build ABS Part using Electroless Deposition Method. Journal of Manufacturing Processes, Volume 19, pp. 22 31

Equbal, A., Sood, A.K., Shamim, M., 2015. Rapid Tooling: A Major Shift in Tooling Practice. Journal of Manufacturing and Industrial Engineering, Volume 14(3-4), pp.1-9

Hanna, F., Hamid, Z.A., Aal, A.A., 2004. Controlling Factors Affecting the Stability and Rate of Electroless Copper Plating. Materials Letters, Volume 58(1-2), pp. 104-109

Hong, S.W., Shin, C.-H., Park, J.-W., 2002. Palladium Activation of $\mathrm{TaN}_{\mathrm{x}}$ Barrier Films for Autocatalytic Electroless Copper Deposition. Journal of The Electrochemical Society, Volume 149(1), pp. 85-88 
Kuzmik, J.J., Mallory, G.O., Hajdu, J.B., 1990. Electroless Plating: Fundamentals and Applications, The American Electroplaters and Surface Finishers Society: Orlando, FL, USA

Li, D., Yang, C.-L., 2009. Acidic Electroless Copper Deposition on Aluminium-seeded ABS Plastics. Surface and Coatings Technology, Volume 203(23), pp. 3559-3568

Lustraflex material safety data sheet, 2009. LEATHERTONE plastic supply, pp. 1-5

Nicolas-Debarnot, D., Pascu, M., Vasile, C., Poncin-Epaillard, F., 2006. Influence of the Polymer Pre-treatment before its Electroless Metallization. Surface and Coatings Technology, Volume 200(14-15), pp. 4257-4265

O'Kelly, J.P., Mongey, K.F., Gobil, Y., Torres, J., Kelly, P.V., Crean, G.M., 2000. Room Temperature Electroless Plating Copper Seed Layer Process for Damascene Interlevel Metal Structures. Microelectronic Engineering, Volume 50(1-4), pp. 473-479

Radulescu, F., Miller, P., Cunnane, L., Harris, M., Lam, H., Bowers, C., 2002. Complete Sputtering Metallization for High-volume Manufacturing. III-Vs Rev, Volume 15(4), pp. 4245

Sood, A.K., Ohdar, R.K., Mahapatra, S.S., 2009. Improving Dimensional Accuracy of Fused Deposition Modeling Processed Part using Grey Taguchi Method. Materials \& Design, Volume 30(10), pp. 4243-4252

Sood, A.K., Ohdar, R.K., Mahapatra, S.S., 2010. Parametric Appraisal of Mechanical Property of Fused Deposition Modelling Processed Parts. Materials \& Design, Volume 31(1), pp. 287-295

Vagramyan, T.A., 1970. Electrodeposition of Alloys: Mechanism of Simultaneous Reaction of Metal Ions, Israel Program of Scientific Translation Ltd, Jerusalem.

Wang, G., Li, N., Li, D., 2007. Effect of Pd Ions in the Chemical Etching Solution. Journal of University Science and Technology, Volume 14(3), pp. 286-289

Xu, C., Wu, G., Liu Z., Wu, D., Meek, T.T., Han, Q., 2004. Preparation of Copper Nano Particles on Carbon Nanotubes by Electroless Plating Method. Materials Research Bulletin, Volume 39(10), pp. 1499-1505 